\title{
Qualitative Assessment of Monomer Ratios in Putative Ionic Terpolymer Samples by Electrospray Ionization Mass Spectrometry with Collision-Induced Dissociation
}

\author{
Benjamin S. Prebyl, Jeremi D. Johnson,* Albert A. Tuinman, \\ Shaolian Zhou, ${ }^{+}$and Kelsey D. Cook \\ Department of Chemistry, University of Tennessee, Knoxville, Tennessee, USA
}

Collision-induced dissociation in the source of an electrospray (ES) mass spectrometer was employed to characterize putative samples of the ionic terpolymer poly(styrene sulfonate-coacrylic acid-co-2-acrylamido-2-methyl-1-propanesulfonic acid). Qualitative and semi-quantitative information about the monomer content was quickly obtainable from ES spectra, and indicated that some samples contained little or none of one or two expected comonomers. For two representative samples, confirmatory nuclear magnetic resonance (NMR) data were acquired. The NMR experiments required sample clean-up (to remove additives) and long acquisition times (up to $720 \mathrm{~min}$ ) for ${ }^{13} \mathrm{C}$ NMR. Cleanup also improved the ES results, providing better agreement with the NMR data. However, qualitative and semi-quantitative information was obtainable by ES (but not by NMR) without the cleanup step. Full quantitation of monomer ratios would require suitable standards, but even without such standards the ES measurements provide a rapid ( $<1 \mathrm{~min})$ means for differentiating these samples (e.g., for process or quality control). (J Am Soc Mass Spectrom 2002, 13, 921-927) () 2002 American Society for Mass Spectrometry

$\mathrm{T}$ The relative amounts of different monomers incorporated into a copolymer can dramatically affect both the chemical and physical properties of the polymer product-a primary motivation for the commercial synthesis of copolymers. Assessment of the monomer ratio is therefore important for assurance of product performance, but such assessment can be challenging. Indirect insight can be obtained from polymerization feed ratios (initial concentrations), monomer reactivities, analysis of residual free monomer after copolymerization, and/or through mathematical kinetics modeling [1,2]. More direct evidence can be obtained from spectroscopic methods such as infrared (IR), nuclear magnetic resonance (NMR), and mass spectrometry (MS) [3-9]. In light of their varying sensitivities and vulnerabilities to interferences, these spectroscopic methods are generally more complementary than competitive.

For polymers of relatively low mass and low polydispersity, MS can provide information about both mo-

Published online June 13, 2002

Address reprint requests to Dr. K. D. Cook, Department of Chemistry, University of Tennessee, Knoxville, TN 37996-1600, USA. E-mail: kcook@utk.edu

*Current address: Department of Chemistry, University of Georgia, Athens, GA 30602-2556.

${ }^{+}$Current address: Covance, Inc., Madison, WI 53707. lecular weight distribution (MWD) and structure [1015]. When polydispersity exceeds about 1.2 , reduced ionization, sampling, and/or detection of heavier oligomers generally result in underestimation of both number-average $\left(\mathrm{M}_{\mathrm{n}}\right)$ and (especially) weight-average $\left(\mathrm{M}_{\mathrm{w}}\right)$ molecular weight [6]. For materials of high mass (typically above a few thousand Da), instrumental ability to resolve specific oligomers usually deteriorates, also compromising quantitation capabilities [16]. In such instances, pyrolysis MS and pyrolysis gas chromatography MS have been used to derive structural information, including monomer identification [3, 4 , 17]. More controlled depolymerization ("unzipping" to the monomer) using ion beam bombardment has also been reported [18, 19].

Many ionization/sampling techniques have been used in polymer MS [3, 4, 8, 10-15]. Electrospray (ES) has proven to be particularly useful for materials that readily form ions in solution [20]. The multiple charging associated with ES is both a boon and a bane in this context. It reduces mass-to-charge $(\mathrm{m} / \mathrm{z})$ ratios, thereby extending the utility of quadrupoles and other common analyzers with limited $\mathrm{m} / \mathrm{z}$ ranges. At the same time, the convolution of charge distributions with MWDs, isotope envelopes, and (for copolymers) distributions of monomer ratios can result in complex, unresolved, and often uninterpretable spec- 


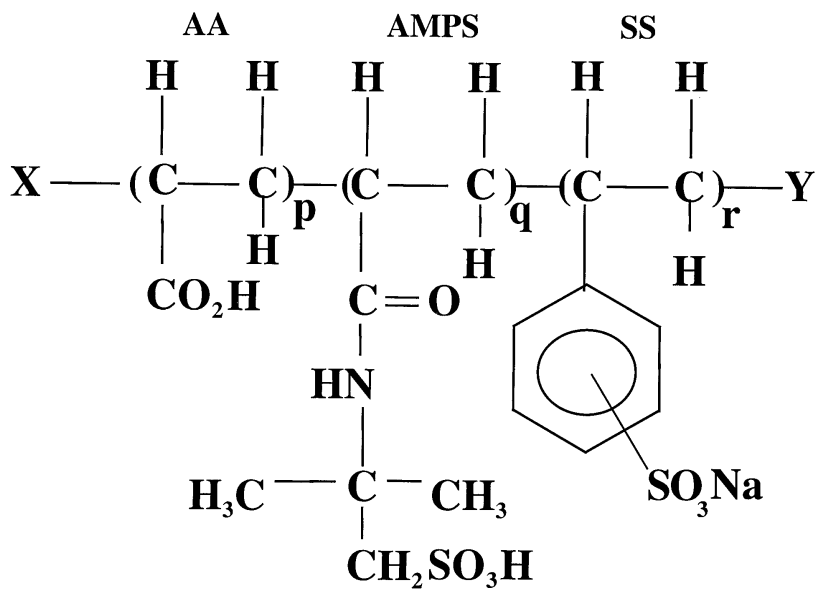

Figure 1. Generic structure of the terpolymers studied. $X$ and $Y$ represent end-groups. $\mathrm{p}, \mathrm{q}$, and $\mathrm{r}$ represent relative monomer content but the figure is not meant to indicate sequence information.

tra. The question arises of whether useful information (e.g., about the monomers comprising a copolymer) can be derived from such complex spectra. We were confronted with this challenge when asked to differentiate monomer ratios among a group of eight samples of a water-soluble polymer (nominal MW $2000-20,000)$ used as a scale inhibitor in various coolant products. The samples putatively shared the structure of Figure 1, but they were derived from different sources, and provided grossly different performance. Since their aqueous matrix included dyes and other additives, it was unclear whether the differences were structural or compositional. Prior efforts aimed at structural characterization by IR and NMR spectroscopies had not been successful, and the acidic functional groups made the polymers appear to be well-suited to analysis by negative ion ES MS. This report describes efforts to derive insight into relative monomer ratios by comparison of the insource collision-induced dissociation (CID) ES mass spectra of these materials. High-resolution proton and ${ }^{13} \mathrm{C}$ NMR data for two samples are included for comparison.

\section{Experimental}

An industrial colleague provided the terpolymer samples (Sample 1-Sample 8) from various manufacturers as aqueous solutions $(\sim 50 \% \mathrm{w} / \mathrm{w})$ with dyes and other additives. As our aim was only to establish whether the polymer constituents differed from one another, specifics of their origin were not pursued and are not reported. Poly(styrene sulfonate), sodium salt (poly-SS; 1600 nominal molecular weight) was obtained from Pressure Chemical Company (Pittsburgh, PA). The remaining reagents were obtained from Aldrich (Milwaukee, WI): HPLC-grade methanol and water; reagent grade $\mathrm{NaOH}$; reagent grade styrene sulfonate (SS) sodium salt; 2-acrylamido-2-methyl-1-propanesulfonic acid (99\%) (AMPS); acrylic acid (99\%) (AA); poly-AMPS $\left(2 \times 10^{6}\right.$ nominal molecular weight); and poly-AA (2000 nominal molecular weight, $65 \mathrm{wt}$. \% solution in water). Except as noted below, all chemicals were used as received from the supplier.

Liquid chromatographic clean-up of polymer Samples 1 and 7 was performed using a $40 \times 2.5 \mathrm{~cm}$ column packed to a height of $\sim 38 \mathrm{~cm}$ with BioGel P-4 from Bio-Rad Labs (Hercules, CA). The void volume of the column was estimated to be $\sim 140 \mathrm{~mL}$. Samples to be purified were first dried by lyophilization. Approximately $0.20 \mathrm{~g}$ of the resulting powder was dissolved in $1 \mathrm{~mL}$ of water and loaded onto the column, which was then gravity-eluted with water (average flow rate 0.8 $\mathrm{mL} / \mathrm{min}$ ). After $90 \%$ of the estimated void volume $(\sim 120 \mathrm{~mL})$ had passed, 30 separate $10 \mathrm{~mL}$ fractions were collected in order to facilitate the freeze-drying process. The total elapsed time for the separation was therefore $\sim 6 \mathrm{~h}$, including $\sim 3.5 \mathrm{~h}$ for collection of the 30 fractions. Material sufficient for NMR analysis $(\sim 0.12 \mathrm{~g})$ was recovered in fractions 3-6, which were collected within $40 \mathrm{~min}$ after the first appearance of polymer (fraction 3). The later fractions (7-30) were pooled and gave proton NMR results similar (within error bars) to those for the earlier fractions. Total solids recovery was roughly $90 \%(0.18 \mathrm{~g})$ for Sample 1 and $85 \%(0.17 \mathrm{~g})$ for Sample 7.

Mass spectra were obtained using a Quattro II (Micromass, Manchester, UK) triple quadrupole mass spectrometer equipped with a standard ES source and a coaxial probe. Preliminary scans of the entire mass range (up to $4000 \mathrm{Da}$ ) were used to select the appropriate ranges for subsequent multi-channel acquisition (MCA) scans. MCA spectra acquired over the selected region (20-30 scans per spectrum) were background-subtracted using a solvent blank. The intensity of the monoisotopic peak was used for ratio calculations. Because of the acidic nature of the monomers, experiments employed the negative ion mode (capillary $-2500 \mathrm{~V}$, counterelectrode $-500 \mathrm{~V}$ ). The cone voltage $(\mathrm{CV})$ was $-20 \mathrm{~V}$ for low energy ("low-CV") experiments and $-100 \mathrm{~V}$ for high-energy ("high-CV") experiments. MS/MS experiments employed a collision energy (CE) of 20-25 V, with nominally $4 \times 10^{-3}$ torr Ar collision gas in the second (RF only) quadrupole. Nitrogen was used for the drying and nebulizing gases at flow rates of 300 and $20 \mathrm{~L} / \mathrm{h}$, respectively. Source temperature was maintained at $80^{\circ} \mathrm{C}$. Monomer and polymer samples were prepared at a concentration of $19.1 \mu \mathrm{g} / \mathrm{ml}$ (except as noted for calibration curves) in 50/50 ( $\mathrm{vol} / \mathrm{vol}$ ) methanol/water containing $75 \mu \mathrm{M}$ sodium hydroxide (to facilitate ionization of weakly acidic monomers). Monomer calibration curves were acquired over the ranges 0.05 to $2 \mu \mathrm{M}$ for SS and AMPS, and 0.03 to 150 $\mathrm{nM}$ for AA. Limits of detection (LOD's) were estimated using sensitivities from these curves to estimate the concentration providing a signal equal to twice the standard deviation of the corresponding 
Table 1. Experimental conditions for NMR experiments

\begin{tabular}{lcc}
\hline & Proton & Carbon-13 \\
\hline \hline Frequency $(\mathrm{Hz})$ & 599.76 & 150.82 \\
Acetone reference peaks $(\mathrm{ppm})^{\mathrm{a}}$ & 2.22 & $30.89,215.94$ \\
$90^{\circ}$ Pulse width $(\mu \mathrm{sec})$ & 8.4 & 20.4 \\
Transients acquired & 16 & 12000 \\
Spectral width $(\mathrm{Hz})$ & 8000 & 50000 \\
Relaxation delay $(\mathrm{sec})$ & 10 & 10 \\
2-D Coupling constant $(\mathrm{Hz})$ & $140^{\mathrm{b}}$ & $8^{\mathrm{c}}$ \\
Repetitions per 2-D increment & $16^{\mathrm{b}}$ & $32^{\mathrm{c}}$ \\
Total acquisition time $(\mathrm{min})$ & 0.5 & 720 \\
\hline
\end{tabular}

${ }^{a}$ vs. tetramethylsilane [25]

${ }^{b}$ Heteronuclear single quantum coherence (HSOC).

cHeteronuclear multiple-bond correlation (HMBC).

blank ( $2 \sigma$ LOD). Samples were infused directly into the source at $5 \mu \mathrm{L} / \mathrm{min}$ using a Harvard Apparatus (South Natik, MA) model 22 syringe pump.

NMR of $3-8 \%(w / v o l)$ copolymer solutions prepared by dissolving lyophilized and purified polymer in $\mathrm{D}_{2} \mathrm{O}[21,22]$ along with $\sim 0.4 \%$ ( $\mathrm{vol} / \mathrm{vol}$ ) acetone (internal reference) were recorded with a Varian (Palo Alto, CA) Inova $600 \mathrm{MHz}$ spectrometer at $298 \mathrm{~K}$ using a $5 \mathrm{~mm}$ triple resonance probe. Experimental parameters are summarized in Table 1. One min acquisitions gave signal:noise ratios $(\mathrm{S} / \mathrm{N})$ $>40$ for proton spectra. ${ }^{13} \mathrm{C}$ NMR acquisition times were established by acquiring until a $\mathrm{S} / \mathrm{N}$ of at least 5 was obtained for the peaks used to calculate the area ratios. Gated decoupling was used to minimize the nuclear Overhauser effect (NOE).

Cited uncertainties and error bars are derived from propagation of error calculations based on the standard deviation for triplicate analysis of a single sample (MS), or single analysis of each of three replicate samples (NMR). These reflect the differences in precision of the experimental methods employed.

\section{Results and Discussion}

Figure 2a presents a typical ES mass spectrum obtained at low cone voltage $(\mathrm{CV}=-20 \mathrm{~V}$; little or no in-source CID) from a terpolymer of the general formula given in Figure 1. As anticipated, the convolution of distributions (charge, mass, monomer ratio, adducting ions, and isotopes) results in an unresolved polymer envelope (here peaking around $\mathrm{m} / \mathrm{z} 400$ ) that defies full interpretation. The position and detailed shape of the envelope differed reproducibly from sample to sample, but the general appearance as an unresolved envelope was invariant for the eight terpolymer samples. The envelope was absent from the solvent blank, unlike the low-mass peaks (below $\mathrm{m} / \mathrm{z} \sim 325$ ) arising from water and/or methanol clusters at low CV. Significantly, there are no prominent peaks in this low-CV polymer spectrum attributable to the constituent monomers. In contrast, peaks corresponding to the conjugate base ([M $\mathrm{H}]^{-}$) of each acid are dominant in negative-ion spectra

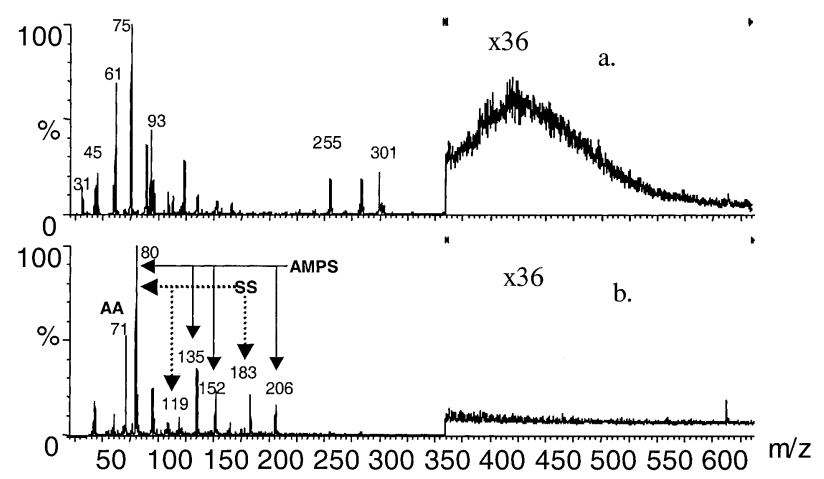

Figure 2. ES mass spectra of a terpolymer (Sample 1 from Figure 3) of the general formula given in Figure 1 obtained with (a) $\mathrm{CV}=$ $-20 \mathrm{~V}$ and (b) $\mathrm{CV}=-100 \mathrm{~V}$. In (b), peaks associated with the individual monomers are mass labeled and the associated monomer is indicated for each. The peak at $\mathrm{m} / \mathrm{z} 80$ derives contributions from both SS and AMPS.

of the individual monomers at low $\mathrm{CV}$, giving strong signals at $m / z 71,183$, and 206 for AA, SS, and AMPS, respectively (Table 2 ). The monomers fragment at high $\mathrm{CV}$, resulting in base peaks at $m / z 80$ for SS and AMPS, and at $m / z 27$ for AA (Table 2). One or more of these monomer-related peaks also become prominent in polymer spectra (at the expense of the unresolved envelope) when the cone voltage is changed to $-100 \mathrm{~V}$ (Table 2 and Figure 2b). In this case, the monomers and their fragments apparently arise as a result of collisioninduced depolymerization in the ES source (see below). The viability of this fragmentation mechanism was confirmed by MS/MS of polymeric precursor ions (Table 2).

Figure 3 reports the relative intensity of each monomer ion $(\mathrm{m} / \mathrm{z} 71,183$, and 206) as a fraction of the total monomer ion intensity [\% $\Sigma=i_{m / z} \times /\left(i_{m / z} 71+\right.$ $\left.i_{m / z} 183+i_{m / z} 206\right)$, where $i$ designates the intensity at the subscripted $\mathrm{m} / \mathrm{z}$ in the high-CV spectrum] for each of the eight target samples. The values differ markedly among the eight samples; all three ions are evident only for Sample 1. The straightforward interpretation is that the monomer ratios differ radically from one sample to another; indeed, only Sample 1 appears to be a true terpolymer, while the others appear to be homopolymers (Samples 2, 4, and 5) or binary copolymers of AA and AMPS (Samples 3, 6, 7, and 8). However, quantitation of ES spectra is never straightforward, especially (as here) in the absence of well-characterized standards. For example, CID spectra like Figure $2 b$ are vulnerable to various suppression effects, such as gas-phase ionmolecule reactions that can attenuate negative-ion signals for stronger gas-phase bases. Corroboration was therefore sought from NMR.

\section{NMR Assessment of Monomer Ratios}

In principle, NMR should provide a suitable alternative methodology for assessment of monomer ratios. However, as noted above, the colleague providing the poly- 
Table 2. Relative intensities for monomer and fragment ions in ES mass spectra of the indicated samples under high ( $-100 \mathrm{~V})$ and low $(-20 \mathrm{~V})$ cone voltage conditions

\begin{tabular}{|c|c|c|c|c|c|c|c|c|c|}
\hline \multirow[b]{2}{*}{ Sample } & \multirow{2}{*}{$\begin{array}{c}\text { Cone } \\
\text { voltage }\end{array}$} & \multicolumn{7}{|c|}{ Relative intensity $(\%)$ at indicated $\mathrm{m} / \mathrm{z}$} & \multirow{2}{*}{$\begin{array}{c}\text { Base peak } \\
\text { intensity }(\mathrm{m} / \mathrm{z})\end{array}$} \\
\hline & & 71 & 80 & 119 & 135 & 152 & 183 & 206 & \\
\hline \multirow[t]{2}{*}{$\overline{\mathrm{AA}}$} & High & & & & & & & & 4.4 E 5(89) \\
\hline & Low & 100 & & & & & & & $7.2 \mathrm{E} 6(71)$ \\
\hline \multirow[t]{2}{*}{ SS } & High & & 100 & $10 \pm 5$ & & & $19 \pm 5$ & & $1.5 \mathrm{E} 8(80)$ \\
\hline & Low & & & $10 \pm 5$ & & & 100 & & $4.5 \mathrm{E} 7(183)$ \\
\hline \multirow[t]{2}{*}{ AMPS } & High & & 100 & & $3 \pm 3$ & & & $9 \pm 4$ & $1.2 \mathrm{E} 8(80)$ \\
\hline & Low & & & & $3 \pm 2$ & $28 \pm 2$ & & 100 & 4.7E6(206) \\
\hline \multirow[t]{3}{*}{ Poly AA } & High & $40 \pm 4$ & & & & & & & 6.7E5(113) \\
\hline & Low & $3 \pm 2$ & & & & & & & $5.0 \mathrm{E} 5(257)$ \\
\hline & Low $^{1}$ & $4 \pm 3$ & & & & & & & 4.1E4(169) \\
\hline \multirow[t]{3}{*}{ Poly SS } & High & & 100 & $55 \pm 4$ & & & $73 \pm 5$ & & $1.2 \mathrm{E} 8(80)$ \\
\hline & Low & & & & & & $5 \pm 3$ & & 7.0E7(290) \\
\hline & Low $^{2}$ & & $7 \pm 4$ & & & & 100 & & $9.4 \mathrm{E} 5(183)$ \\
\hline \multirow[t]{3}{*}{ Poly AMPS } & High & & $47 \pm 1$ & & $67 \pm 3$ & $26 \pm 9$ & & 100 & 1.5E6(206) \\
\hline & Low & & & & & & & & 7.9E5(700) \\
\hline & Low $^{3}$ & & $53 \pm 3$ & & $35 \pm 3$ & $40 \pm 6$ & & $32 \pm 2$ & $8.2 \mathrm{E} 4(762)$ \\
\hline \multirow{2}{*}{$\begin{array}{l}\text { Terpolymer } \\
\text { (Sample 1) }\end{array}$} & High & $54 \pm 4$ & 100 & $10 \pm 4$ & $36 \pm 6$ & $24 \pm 5$ & $21 \pm 7$ & $16 \pm 5$ & $5.9 \mathrm{E} 6(80)$ \\
\hline & Low & & & & & & & & $4.8 \mathrm{E} 6(75)$ \\
\hline
\end{tabular}

${ }^{1} \mathrm{MS} / \mathrm{MS}$ of precursor at $\mathrm{m} / \mathrm{z} 257\left[\mathrm{AA}_{3}+42-\mathrm{H}\right]^{-}$.

${ }^{2} \mathrm{MS} / \mathrm{MS}$ of precursor at $\mathrm{m} / \mathrm{z} 340\left[\mathrm{SS}_{5}+58+2 \mathrm{Na}\right]^{3-}$.

${ }^{3} \mathrm{MS} / \mathrm{MS}$ of precursors in a window $\sim 3 \mathrm{~m} / \mathrm{z}$ wide centered at $\mathrm{m} / \mathrm{z} 685$. It was not possible to resolve individual oligomers of this high-mass polymer, so the first quadruple was detuned to pass ions near the top of the unresolved polymer envelope.

Note: Blank entries indicate signals indistinguishable from background. Uncertainties are the standard deviation of triplicate measurements.

mer samples reported that (low resolution) NMR characterization had not been successful. Using two test samples (Samples 1 and 7) that had been lyophilized and redissolved in $\mathrm{D}_{2} \mathrm{O}$, we found that even with the relatively high-resolution Varian Inova 600 instrument, proton and ${ }^{13} \mathrm{C}$ NMR spectra were too complex to provide clear confirmation of apparent monomer ratios (data not shown).

Suspecting that this complexity was attributable at least in part to the presence of additives, we undertook the lengthy clean-up procedure described in the

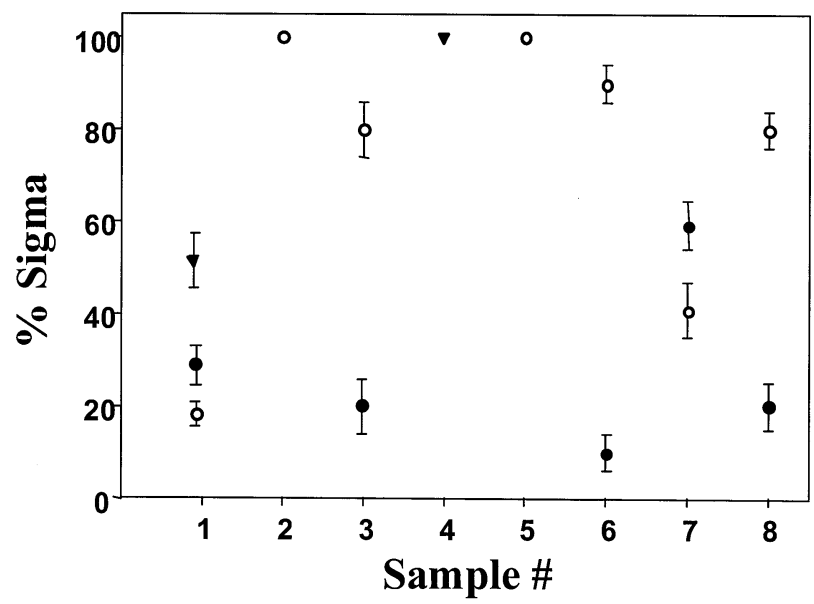

Figure 3. Relative intensity of monomer anions $(\% \Sigma$; see text for definition) illustrating qualitative differences among the ES mass spectra of the eight terpolymers sampled. Symbols: filled circle, open circle, and filled inverted triangle represent AMPS, acrylic acid, and styrene sulfonate, respectively. Error bars represent 1 standard deviation for triplicate analyses.
Experimental section. This removed $\sim 10$ and $15 \%$ of the dry mass of Sample 1 and Sample 7 respectively, and substantially simplified the NMR spectra. Proton NMR spectra of the purified polymers could be obtained relatively quickly ( $<1 \mathrm{~min}$.; Table 1$)$, and could resolve contributions from SS (aromatic protons at 7.3. and $7.7 \mathrm{ppm}$ ) and AMPS (methyl protons at $1.4 \mathrm{ppm}$, and methylene protons adjacent to sulfonate at $3.2 \mathrm{ppm}$ ) in Sample 1. Consistent with the data of Figure 3, the aromatic protons were not detected in the NMR of Sample 7.

Unfortunately, the proton spectra could not provide insight into the AA content of the samples. Resonances attributable to the acid protons of AA are $\mathrm{pH}$-sensitive and broad. Even in spectra of the purified polymers, contributions from backbone protons (1.5-1.8 and 2.0-2.3 ppm) could not be separately assigned to the three constituent monomers; such assignment is precluded by the complexity derived from the wide variety of monomer "triad" orientations of adjacent monomers possible in these random copolymers [1, 21, 22]. This complexity also precludes unambiguous assignment of backbone carbons (33-38 and 44-46 ppm for various $\mathrm{CH}_{2}$ and $\mathrm{CHR}$ carbons, respectively). However, ${ }^{13} \mathrm{C}$ NMR spectra (requiring acquisition time up to $720 \mathrm{~min}$; Table 3) do contain other features characteristic of the individual monomers: carbonyl peaks for AA and AMPS (178.5 and 182.3 ppm, respectively) and aromatic carbons for SS (150-153 ppm). The absence of the latter signals in the ${ }^{13} \mathrm{C}$ spectrum of Sample 7 again confirms the absence of SS from this sample.

More detailed comparison of the normalized inten- 
Table 3. Relative intensities of signals associated with the indicated monomers in NMR and ES spectra of Samples 1 and 7 before ("Orig") and after ("GPC") chromatographic clean-up

\begin{tabular}{|c|c|c|c|c|c|c|c|c|c|c|}
\hline \multirow[b]{2}{*}{ Sample } & \multicolumn{3}{|c|}{$\% A A$} & \multicolumn{3}{|c|}{$\%$ AMPS } & \multicolumn{3}{|c|}{$\%$ SS } & \multirow[b]{2}{*}{$\mathrm{S}_{\mathrm{rel}, \mathrm{MS}}$} \\
\hline & MS & $\begin{array}{c}{ }^{1} \mathrm{H} \\
\mathrm{NMR}\end{array}$ & $\begin{array}{c}{ }^{13} \mathrm{C} \\
\mathrm{NMR}\end{array}$ & MS & $\begin{array}{c}{ }^{1} \mathrm{H} \\
\text { NMR }\end{array}$ & $\begin{array}{c}{ }^{13} \mathrm{C} \\
\text { NMR }\end{array}$ & MS & $\begin{array}{c}{ }^{1} \mathrm{H} \\
\text { NMR }\end{array}$ & $\begin{array}{c}{ }^{13} \mathrm{C} \\
\mathrm{NMR}\end{array}$ & \\
\hline Orig\#1 & $19 \pm 2$ & * & * & $28 \pm 3$ & * & $*$ & $53 \pm 4$ & * & * & $\overline{0.20 \pm 0.08}$ \\
\hline Orig\#7 & $40 \pm 5$ & $*$ & * & $60 \pm 5$ & * & * & 0 & 0 & 0 & $0.53 \pm 0.13$ \\
\hline GPC\#1 & $24 \pm 3$ & * & $11 \pm 4$ & $30 \pm 3$ & $86 \pm 2$ & $82 \pm 8$ & $46 \pm 4$ & $14 \pm 2$ & $7 \pm 4$ & $0.17 \pm 0.07$ \\
\hline GPC\#7 & $73 \pm 4$ & * & $26 \pm 6$ & $27 \pm 3$ & 100 & $74 \pm 6$ & 0 & 0 & 0 & $0.13 \pm 0.03$ \\
\hline
\end{tabular}

* Interference precluded assessment.

Note: Each entry represents intensity relative to the sum of monomer-related intensitites (\% $\Sigma$; see text for details). The final column represents the relative $E S$ sensitivity for AMPS and $A A\left(S_{A M P S}, M S / S_{A A}\right.$, Ms; see Eq 1 for definition), based on the assumption that the corresponding ${ }^{13} C$ NMR data for the purified sample accurately represents the relative abundances.

sities in Table 3 is complicated by the fact that the sensitivities for the different monomers can be predicted with confidence to be roughly equal only for the proton NMR data; the NOE may complicate quantitation of ${ }^{13} \mathrm{C}$ NMR, and matrix effects often distort ES sensitivities. The distortion of ES data is evident, for example, from comparison of the relative signals for AMPS and SS for purified Sample 1; from the ${ }^{1} \mathrm{H}$ NMR data, AMPS:SS is $\sim 6 \pm 1(86 / 14)$, versus $\sim 0.6 \pm$ $0.1(30 / 46)$ for the MS data (uncertainties reflect the propagation of errors). If the NMR data accurately reflect the composition, AMPS must be "underrepresented" in the ES data, by a factor of about 6/0.6 10 \pm 2 . By contrast, the AMPS:SS ratios for proton and ${ }^{13} \mathrm{C}$ NMR data are in reasonable agreement (AMPS:SS $\sim 12 \pm 7$ for ${ }^{13} \mathrm{C}$ NMR, within experimental error of the value for the proton NMR data), suggesting that the NOE may not be extreme in this instance. If the NOE is negligible, the normalized ${ }^{13} \mathrm{C}$ NMR values in Table 3 may be taken to represent the actual monomer ratios in the polymer, providing a benchmark for assessing the self-consistency of the ES results, i.e., the ability to use sensitivities derived from one sample to quantitate another.

Because there was no SS detected for Sample 7, consistency can best be tested by considering AMPS and AA. By analogy to the development above, the "true" AMPS:AA ratio for Sample 1 would be 82/11 $\approx 7.4 \pm 2.8$, based on ${ }^{13} \mathrm{C}$ NMR. From the MS data, the corresponding intensity ratio is only $1.2 \pm 0.2$, indicating in this instance that AMPS is underrepresented by a factor of about $6.0 \pm 2.4(7.4 / 1.2)$. Applying this factor to the MS data for Sample 7 gives a "corrected" concentration ratio of $2.2 \pm 0.9$, within experimental error of the value determined from the ${ }^{13} \mathrm{C}$ NMR data for this data $(2.8 \pm 0.5)$. This indicates that the relative ES sensitivities to AMPS and AA were similar for the two samples, so that one sample could be used as a calibration standard for the other. In an equivalent treatment, we can define the relative ES sensitivities $\left(\mathrm{S}_{\text {rel, }}\right.$ MS) as in Eq 1:

$$
\begin{aligned}
\mathrm{S}_{\mathrm{rel}, \mathrm{MS}} & \equiv\left(\mathrm{S}_{\mathrm{AMPS}, \mathrm{MS}}\right) /\left(\mathrm{S}_{\mathrm{AA}, \mathrm{MS}}\right) \\
& =\left(i_{\mathrm{AMPS}, \mathrm{MS}} / i_{\mathrm{AA}, \mathrm{MS}}\right) /\left(i_{\mathrm{AMPS}, \mathrm{NMR}} / i_{\mathrm{AA}, \mathrm{NMR}}\right)
\end{aligned}
$$

where the intensities (i) correspond to the entries in Table 3. Of the $S_{\text {rel, }}$ ss values calculated for each of the four samples (last column of Table 3), three agree within experimental error. The "outlier" is the value obtained for unpurified Sample 7, suggesting that this sample (but not Sample 1) contained a significant MS interferent which was removed by the purification process.

Significantly, no signal appeared in the proton NMR spectra of either sample in the region of 5.2-6.7 ppm [23], where vinylic protons from the unreacted monomers would be expected to appear. Detection of such residual monomer contaminants is often an important part of polymer characterization. In light of the wide dynamic range, high sensitivity, and sometimes undesirable selectivity of mass spectrometry, it became of interest to assess whether the monomer signals observed in Figure $2 \mathrm{~b}$ might have

Table 4. Limit of detection $(\mathrm{nM})$ for the indicated monomer in electrospray mass spectra obtained at high $(-100 \mathrm{~V})$ and low $(-20 \mathrm{~V})$ cone voltage, with and without the presence of $19.1 \mu \mathrm{g} / \mathrm{mL}$ terpolymer (Sample 1)

\begin{tabular}{lccrr}
\hline & \multicolumn{2}{c}{ Without terpolymer } & \multicolumn{2}{c}{ With terpolymer } \\
\cline { 2 - 4 } Monomer & $\mathrm{CV}=-20 \mathrm{~V}$ & $\mathrm{CV}=-100 \mathrm{~V}$ & $\mathrm{CV}=-20 \mathrm{~V}$ & $\mathrm{NV}=-100 \mathrm{~V}$ \\
\hline \hline AA & $0.04 \pm 0.01$ & $\mathrm{~N} / \mathrm{A}^{1}$ & $15 \pm 2$ & $280 \pm 20$ \\
SS & $15 \pm 1$ & $40 \pm 2$ & $24 \pm 2$ & $80 \pm 10$ \\
AMPS & $10 \pm 2$ & $20 \pm 1$ & $25 \pm 2$ & $\mathrm{C}$ \\
\hline
\end{tabular}

${ }^{1}$ Not detectable above background. 
derived from relatively high sensitivity to relatively low concentrations of residual monomers in the polymer samples, rather than from depolymerization.

\section{Testing for Residual Monomers}

From simple calibration curves for the individual monomers obtained at high CV (data not shown), it can be estimated that roughly $1.6 \pm 0.2$ and $0.9 \pm 0.1 \mu \mathrm{M} \mathrm{SS}$ and AMPS, respectively, would be necessary to account for the signals at $\mathrm{m} / \mathrm{z} 183$ and 206 in Figure $2 \mathrm{~b}$. By comparison, the $2 \sigma$ LOD's for these monomers at low$\mathrm{CV}$ in the presence of Sample 1 are 24 and $25 \mathrm{nM}$ for SS and AMPS, respectively (Table 4); had monomers been present at the $\mu \mathrm{M}$ level, they should have been detected in Figure 2a.

Significantly, a corresponding comparison for AA was not possible; fragmentation of this monomer (primarily loss of $\mathrm{CO}_{2}$ to form $\mathrm{m} / \mathrm{z} 27$ ) was so extensive at high-CV that no significant signal was detectable above the background at $m / z 71$ when sampling monomers, so a high-CV calibration curve could not be constructed for AA. Only the dissipation of energy upon polymer fragmentation enabled detection of the intact AA monomer at $\mathrm{m} / \mathrm{z} 71$ with signal-to-noise appreciably above that of the background at high-CV. We can therefore conclude that all AA monomer-related signals, and most or all SS and AMPS monomer-related signals detected in Figure $2 b$ must have derived from depolymerization.

In the course of the calibration experiments used to estimate the monomer that would have been needed to account for the signals in Figure 2b, LOD's were determined at high- and low-CV, both with and without polymer present. These data are reported in Table 4. As expected, they show that at the higher $\mathrm{CV}$ and in the presence of polymer, the increase in "background" (i.e., the signal derived from depolymerization) and concomitant noise in the system resulted in a higher (poorer) LOD.

\section{Conclusions}

Mass spectrometric assessment of monomer ratios clearly requires independently well-characterized standards. Work in progress [24] involving binary copolymers of SS and maleic acid (MA) suggests that simple monomer mixtures will not provide good calibration standards; polymer standards generally will be needed for reliable quantitation. Nonetheless, the correlation between the NMR and MS data of Table 3 indicates that data like that of Figure 3 reflect differences among samples. MS may benefit from sample clean-up, but for these samples it was not essential. NMR provided equivalent or better information, but required both time-consuming clean-up and long acquisition times (up to $720 \mathrm{~min}$.). In the long run, information may be extractable from data like that of Figure 2a; deriving tools for interpretation of such spectra remains a goal of our work.

\section{Acknowledgments}

Support for this work was provided by the University of Tennessee Measurement and Control Engineering Center (an NSF Industry/University Cooperative Research Center, grant EEC-9908040), and by the National Science Foundation (grant EEC-9634522). The authors gratefully acknowledge Karen Welch's assistance with NMR instrumentation.

\section{References}

1. Czerwinski, W. K. Improved Increments for Characterization of Comonomer Sequencing in Binary Copolymers. Polymer 1997, 38, 1381-1385.

2. Kuchanov, S. I. Principles of the Quantitative Description of the Chemical Structure of Synthetic Polymers. Advances in Polymer Science 2000, 152, 157-201.

3. Smith, P. B.; Pasztor, A. J., Jr.; McKelvy, M. L.; Meunier, D. M.; Froelicher, S. W.; Wang, F. C. Y. Analysis of Synthetic Polymers and Rubbers. Anal. Chem. 1999, 71, 61R-80R.

4. Smith, C. G.; Smith, P. B.; Pasztor, A. J., Jr.; McKelvy, M. L.; Meunier, D. M.; Froelicher, S. W. Analysis of Synthetic Polymers and Rubbers. Anal. Chem. 1995, 67, R97-R126.

5. Westler, W. M.; Ortiz-Polo, G.; Markley, J. L. Two-Dimensional Proton-Carbon-13 Chemical-Shift Correlated Spectroscopy of a Protein at Natural Abundance. J. Magn. Reson. 1984, 58, 354-357.

6. Danis, P. O.; Karr, D. E.; Xiong, Y.; Owens, K. G. Methods for the Analysis of Hydrocarbon Polymers by Matrix-Assisted Laser Desorption/Ionization Time-of-Flight Mass Spectrometry. Rapid Commun. Mass Spectrom. 1996, 10, 862-868.

7. Chen, G.; Cooks, R. G.; Jha, S. K.; Oupicky, D.; Green, M. M. Block Microstructural Characterization of Copolymers Formed from Fluorinated and Non-Fluorinated Alkyl Polyisocyanates Using Desorption Chemical Ionization Mass Spectrometry. Int. J. Mass Spectrom. Ion Processes 1997, 165, 391-404.

8. Shard, A. G.; Volland, C.; Davies, M. C.; Kissel, T. Information on the Monomer Sequence of Poly(lactic acid) and Random Copolymers of Lactic Acid and Glycolic Acid by Examination of Static Secondary Ion Mass Spectrometry Ion Intensities. Macromolecules 1996, 29, 748-754.

9. Beavis, R. C. Matrix-Assisted Ultraviolet Laser Desorption: Evolution and Principles. Org. Mass Spectrom. 1992, 27, 653659.

10. Wilkins, C. L. Mass Spectrometric Analysis of Polymers. Applied Polymer Sci. 2000, 817-827.

11. Scrivens, J. H.; Jackson, A. T. Characterization of Synthetic Polymer Systems. Int. J. Mass Spectrom. 2000, 200, 261-276.

12. Hanton, S. D. Mass Spectrometry of Polymers and Polymer Surfaces. Chem. Rev. (Washington, D. C.) 2001, 101, 527-569.

13. Montaudo, M. S. Recent Advances in the Analysis of Polymers and Copolymers by Mass Spectrometry. Macromol. Symp. 1999, 141, 95-101.

14. Montaudo, G. Mass Spectrometry of Synthetic Polymers. Mere Advances or Revolution? Trends Polym. Sci. (Cambridge, U. K.) 1996, 4, 81-86.

15. Cook, K. D., Ed.; In Encyclopedia of Polymer Science and Engineering; Kroschwitz, J., Ed.; 1987; Vol. IX, pp. 319-356.

16. Rashidzadeh, H.; Guo, B. Use of MALDI-TOF to Measure Molecular Weight Distributions of Polydisperse Poly(methyl methacrylate). Anal Chem. 1998, 70, 131-135.

17. Bart, J. C. J. Polymer/Additive Analysis by Flash Pyrolysis Techniques. J. Anal. Appl. Pyrolysis 2001, 58/59, 3-28.

18. Fragala, M. E.; Compagnini, G.; Torrisi, L.; Puglisi, O. Ion Beam Assisted Unzipping of PMMA. Nucl. Instrum. Methods Phys. Res. Sect. B 1998, 141, 169-173. 
19. Lattimer, R. P. Fast Atom Bombardment Mass Spectrometry of Polyglycols. Int. J. Mass Spectrom. Ion Processes 1984, 55, 221-232.

20. Fenn, J. B.; Mann, M.; Meng, C. K.; Wong, S. F.; Whitehouse, C. M. Electrospray Ionization-Principles and Practice. Mass Spectrom. Rev. 1990, 9, 37-70.

21. Travas-Sejdic, J.; Easteal, A. Study of Free-Radical Copolymerization of Acrylamide with 2-Acrylamido-2-Methyl-1-Propane Sulfonic Acid. J. Appl. Polym. Sci. 2000, 75, 619-628.

22. Rangaraj, A.; Vangani, V.; Rakshit, A. K. Synthesis and Characterization of Some Water-Soluble Polymers. J. Appl. Polym. Sci. 1997, 66, 45-56.
23. SBDS, Integrated Spectral Data Base System for Organic Compounds (2001), http://www.aist.go.jp/RIODB/SDBS/ menu-e.html retrieved September 5, 2001.

24. Prebyl, B.; Cook, K. D.; Johnson, J. Electrospray Mass Spectrometry with Collison-Induced Dissociation for Assessment of Monomer Ratios in Copolymers. Proceedings of the 49th Annual Conference on Mass Spectrometry and Allied Topics, Chicago, IL, May, 2001.

25. Gottlieb, H. E.; Kotlyar, V.; Nudelman, A. NMR Chemical Shifts of Common Laboratory Solvents as Trace Impurities. J. Org. Chem. 1997, 62, 7512-7515. 